\title{
Order from disorder in closed systems via time reversal violation
}

\author{
T. Goldman and D.H. Sharp \\ Theoretical Division \\ Los Alamos National Laboratory \\ Los Alamos, NM 87545 USA
}

\begin{abstract}
Definitions of entropy usually assume time-reversal $(\mathrm{T})$ invariance of interactions, yet microscopically $\mathrm{T}$ is known to be violated. We present a detailed computational example of (uncharged) particle species separation (Maxwell demon) using an interaction that violates both parity (P) and $\mathrm{T}$ so that PT is preserved, consistent with the CPT invariance required in quantum field theory $(\mathrm{C}$ is charge conjugation). This illustrates how T-violating forces can produce more organized states from disorganized ones, contrary to expectations based on increase of entropy. We also outline several scenarios in which T-violating forces could lead to an organized state in the early Universe, starting from a still earlier disorganized state.
\end{abstract}


PACS: 11.30.Er, 98.80.-k, 98.80.Cq

\section{INTRODUCTION}

A central problem of statistical mechanics has been to understand the monotonic increase of thermodynamic entropy in the presence of time-reversal-invariant dynamics. The resolution of this problem is based on the observation that the number of available states with higher entropy, for all but the simplest systems, far exceeds those accessible with the same or lower entropy. An organized state corresponds to one in which a small subset of the available states is preferentially occupied. The result is a probabilistic conclusion that on average entropy increases uniformly, although local fluctuations are possible in principle. (An instructive example from everyday experience has been given by T.D. Lee[1].)

Here we discuss a related question: If the dynamics violates time reversal invariance, is it still true that entropy must increase? Or, with the defined arrow of time, is it possible that entropy must in fact decrease, at least for sufficiently large T-violation?

If true, the latter possibility raises some intriguing questions when applied to the early Universe: If conventionally-defined entropy can decrease when T-violation is sufficiently large, there appear to be mechanisms that could lead to the minimal entropy state assumed in the Big Bang hypothesis, even if the universe was in a high entropy (disorganized) state at times prior to the Big Bang. Several such mechanisms are discussed here, along with their bearing on such matters as baryon asymmetry.

\section{EXAMPLE 1: SCATTERING OF DISTINGUISHABLE PARTICLES IN 1 DI- MENSION}

The example we discuss here is from classical physics, but is chosen so as to be consistent with the CPT theorem [3], which is one of the basic constraints of conventional quantum field theory. The CPT theorem states that the combination of charge conjugation, parity inversion and time reversal operations leaves the world Lagrangian invariant.

Consider two distinguishable types of classical particle A and B moving in a $1+1$ dimen- 
sional manifold. If the particles are uncharged, the overall Lorentz symmetry constraint in a flat space-time requires that the combination of parity $(\mathrm{P})$ and time-reversal $(\mathrm{T})$ is an invariance of the dynamics. Combined PT invariance is necessary to satisfy the requirement that all amplitudes be restricted to the proper, orthochronous sector of the Lorentz transformations; P and T need not separately be invariances of the dynamics. Access to a wide range of papers that consider $\mathrm{P}$ and $\mathrm{T}$ violation while preserving $\mathrm{PT}$ can be found at $<$ http: //ptsymmetry.net $>$. (The CPT theorem has not been demonstrated in curved space-times. [4] )

We now introduce an interaction which violates $\mathrm{P}$ and $\mathrm{T}$ separately, but preserves the product. Specifically, we suppose the probability is unity that A, incoming from the left, and $\mathrm{B}$, incoming from the right, reaches the final state in which $\mathrm{A}$ continues to the right and $\mathrm{B}$ continues to the left. (This scattering and all others described below are taken to be perfectly elastic, energy-momentum conserving scatterings. For convenience in our calculations, we will also assume equal masses for the distinguishable particles, so that energy-momentum obtains trivially.) Further, we assume that the amplitude for the other possible final state (A reflected back to the left and B back to the right) vanishes identically. That is,

$$
\begin{aligned}
& P\left(p_{A}>0, p_{B}<0 \rightarrow p_{A}^{\prime}=p_{A}>0, p_{B}^{\prime}=p_{B}<0\right)=1 \\
& P\left(p_{A}>0, p_{B}<0 \rightarrow p_{A}^{\prime}=p_{B}<0, p_{B}^{\prime}=p_{A}>0\right)=0
\end{aligned}
$$

where the values of the momenta are unchanged in the first case, and the values for particles $\mathrm{A}$ and $\mathrm{B}$ are exchanged in the second.

However, for the parity reversed initial state ( A incoming from the right and B incoming from the left), we suppose that the probability is unity for scattering to the final state in which A reflects back to the right and B reflects back to the left and that there is zero probability for each particle to continue forward. That is,

$$
\begin{aligned}
& P\left(p_{A}<0, p_{B}>0 \rightarrow p_{A}^{\prime}=p_{B}>0, p_{B}^{\prime}=p_{A}<0\right)=1 \\
& P\left(p_{A}<0, p_{B}>0 \rightarrow p_{A}^{\prime}=p_{A}<0, p_{B}^{\prime}=p_{B}>0\right)=0
\end{aligned}
$$

These probabilities clearly maximally violate $\mathrm{P}$ and $\mathrm{T}$ separately. On applying both transformations, it is also apparent that the PT product is nonetheless an invariant; we take all of the $\mathrm{AA}$ and $\mathrm{BB}$ forward and backward scattering probabilities to be equal. 
We observe that the equilibrium state of this dynamics is a separation of any initial mixture of $\mathrm{A}$ and $\mathrm{B}$ into a segregated system with only particles of type $\mathrm{A}$ to the right and only particles of type B to the left, where the location of the dividing line is determined by the relative numbers of the two particle types. The dynamics has acted as a Maxwell demon[5] to produce a state which has lower entropy (as defined conventionally) than the normally expected one of maximal mixing of the two particle types.

Note, though, that no work can be extracted from the separated system because the particles will not tend to remix unless the dynamics changes. Preservation of the concept that entropy can only increase now clearly requires some different definition of entropy in order to assign the separated system a higher value of the entropy than the fully mixed one. That is, the final state is achieved not due to directed energy flow or externally imposed chemical potential differences, but is an equilibrium different from the one expected with $\mathrm{P}$ - and T-conserving dynamics. It is the fully mixed initial state that is out of equilibrium!

We have verified the conclusions above in this 1+1-dimensional case by means of Monte Carlo simulation programs.

We first wrote a Monte Carlo simulation program with T-conserving dynamics, where all of the probabilities in Eqs.(1,2) are equal. The program populates the phase space with particles $\mathrm{A}$ and $\mathrm{B}$ having equal temperatures and uniformly mixed initial spatial distributions. Scattering is introduced when any two particles propagate into close proximity. We have verified that with the P,T-conserving dynamics the system maintains the expected equilibrium distribution and the two types of particles remain fully mixed, although separation fluctuations of one standard deviation either way quite clearly occur. Following the motion of a few particles chosen at random shows that they do propagate and scatter as expected. Figure 1 displays the uniform spatial mixing (up to fluctuations), temperature equality and phase space distribution in the presence of scattering but in the absence of a T-violating interaction.

We then changed the dynamics so as to satisfy the T-violating conditions described above in Eqs.(1 2): we require that if $\mathrm{A}$ has negative velocity and $\mathrm{B}$ has a positive value, no scattering takes place, but if A has positive velocity and B has negative value, then the particles reflect (in the easiest to understand, equal mass case, satisfying energy-momentum conservation) with $\mathrm{A}$ acquiring the negative velocity of $\mathrm{B}$ and $\mathrm{B}$ the positive one of $\mathrm{A}$. In this case, the initial 

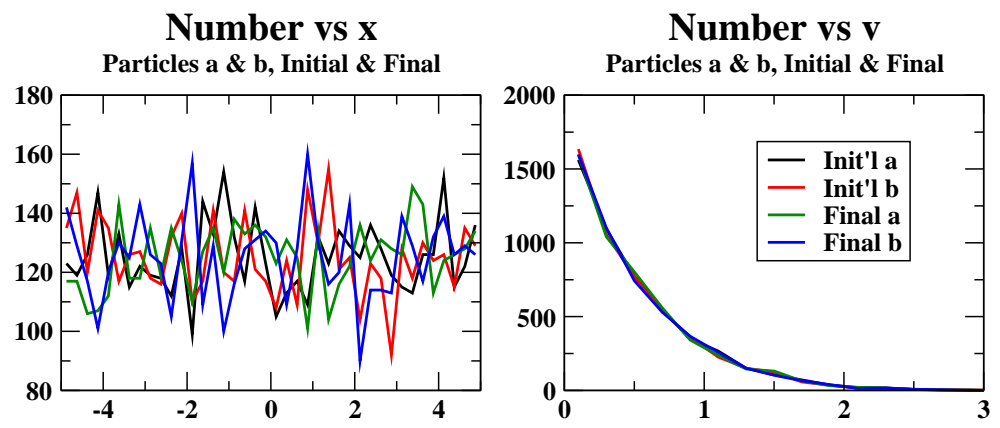

Phase Space

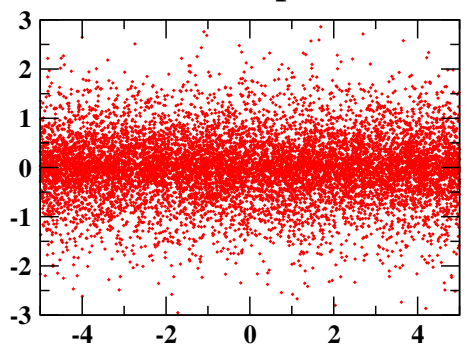

Left-Right a\&b vs Time

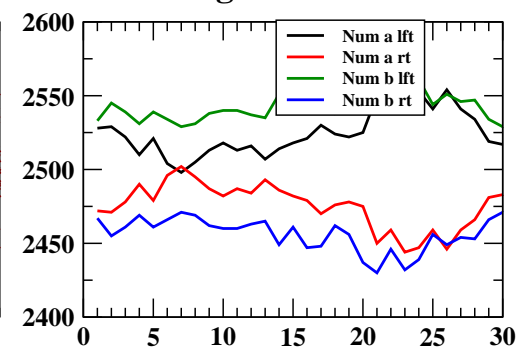

FIG. 1: (Color online) T-conserving distributions and time evolution. a) Upper left panel: Binned initial and final numbers of particles vs. position along line; color coding is the same as in the upper right panel b) which shows the velocity distributions. The phase space distribution, ignoring particle type, is shown in the lower left panel, c). The time variation of the number of particles of each type to the left or right of the center line is shown in the lower right panel, d).

uniformly mixed distribution undergoes the expected segregation, with particles of type B flowing to positive spatial locations and particles of type A flowing to negative ones. The separation occurs quite rapidly for maximal violation. Figure 2 displays the initial uniform spatial mixing (up to fluctuations) and the clear separation of A and B species after the T-violating interactions have been turned on to affect the scattering. Temperature equality is maintained and the phase space distribution, ignoring the particle type, is qualitatively unaltered; the numerical evidence indicates that the phase space volume is conserved. The final state equilibrium is attained after approximately 10 time units.

Our results also show that if we reduce the effective strength of $\mathrm{P}$ and T-violation by limiting the (random) fraction of $\mathrm{AB}$ scatters that violate $\mathrm{P}$ and $\mathrm{T}$ to $5 \%$ (i.e., the zeros on the rhs of Eqs. (1.2) are replaced by 0.95 ) and require the remaining fraction to satisfy these invariances, the same segregation develops. This is shown in Figure 3. (Temperature and phase space 

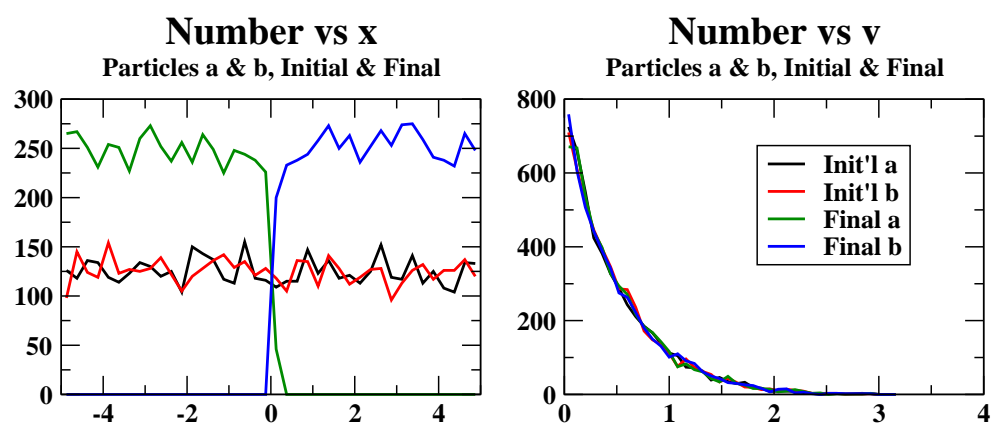

Phase Space

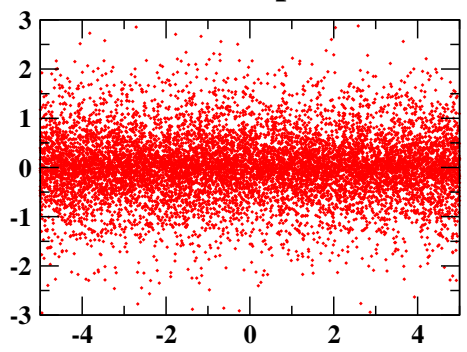

Left-Right a\&b vs Time

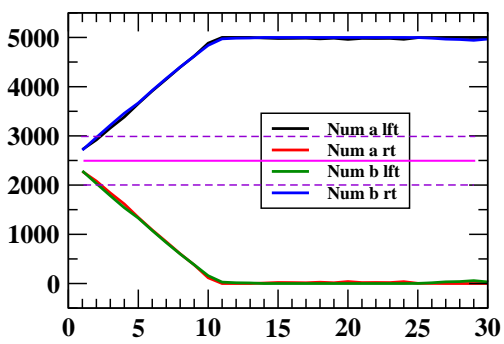

FIG. 2: (Color online) T-violating distributions and time evolution with maximal violation. Panels and color coding as in Fig.1; note different scale in phase space distribution. Upper left panel a) shows initial distributions fully mixed and final distributions well separated except for small central transition region. The dashed lines in the lower right panel d) show the one standard deviation expectation from the number of each species of particle.

distributions are unaffected and so are not repeated.) Note that, as expected, it takes longer for the final state equilibrium to be reached; this is now attained after approximately 15 time units.

The fractional amount of separation in the population increases linearly with time in both cases. Examining the separation before it goes to completion shows an interesting pattern: The extreme positive and negative ends of the line become saturated with particles B and A respectively, but an intermediate, quite mixed, region remains. (Note that this is different from the conventional Maxwell demon picture.) Observation of the track of randomly chosen particles confirms this: An A particle that happens to originate in a negative region tends to remain there, while one that originates in a positive region moves, albeit with unsteady fluctuations, towards the negative region, and vice versa for $\mathrm{B}$ particles. This directly displays the feature that for A-B scattering, the built-in T-violation of the scattering matrix 

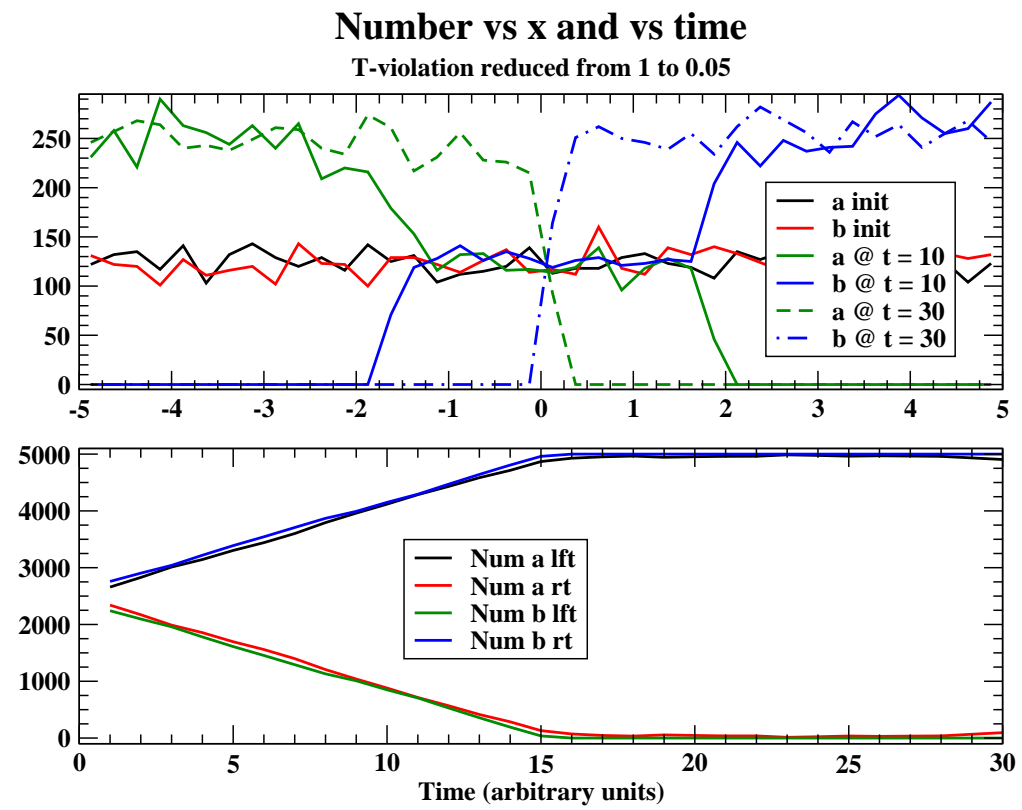

FIG. 3: (Color online) T-violating distributions a) and time evolution b) with reduced violation ( $5 \%$ of maximal). Note the longer time scale for the system to separate vs. that in Fig.2.

causes A particles with positive velocity to be reflected but allows those with negative velocity to proceed unhindered, and conversely for the B particles. A similar phenomenon (termed "rectification") has been observed by Wan et al. in a numerical simulation of bacterial motion in the presence of barriers[2] that reproduces experimental observations of the same system.

\section{EXAMPLE 2: SCATTERING OF PARTICLES IN 3 DIMENSIONS}

One might be concerned that these conclusions are limited to one dimension. To investigate this possibility, we consider a different closed system - this time a sphere within which the two types of particles are confined, and subject to scattering that depends on the sign of the dot product of the velocity of one species with the radial vector of either the other species, or with itself. Such a system will in general exhibit complex motions but, as our computations show, still evolves to a final state with segregation of the two species. See Figs. (4 5,6 .

Another concern that might be raised regarding these calculations is that we have not 


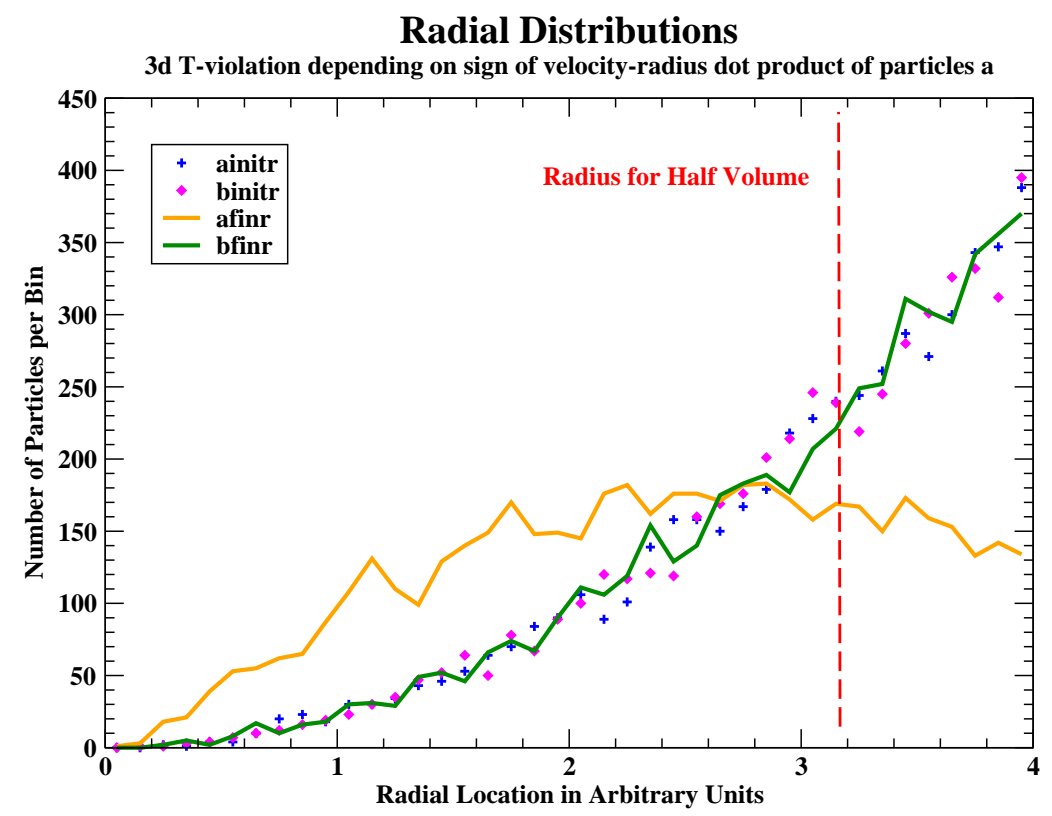

FIG. 4: (Color online) T-violating distributions when the scattering depends on the sign of the dot product of the velocity of one species with its radial vector. The initial radial distributions (ainitr, binitr) are constructed to be uniform. After some time, the species (a) subject to the T-violating scattering has been significantly compacted to the inner half-volume of the reference sphere (afinr) of radius 4 units.

explicitly derived the assumed scattering properties from a Hamiltonian. These properties, however, have been modeled after known results in the weak interactions, for the onedimensional case. There the interaction is $\mathrm{P}$-violating but avoids T-violation by including $\mathrm{C}$-violation, As $\mathrm{C}$ is not available in our model problem, the P-violation directly translates to T-violation as noted earlier. For the three-dimensional case, the $\vec{r} \cdot \vec{p}$ interaction corresponds to scattering in a fixed (T-violating) background electromagnetic field[6]. We note this to confirm that our scattering matrix is derivable from a Hamiltonian; it does not imply that we are dealing with an open system. We conclude, therefore, that our examples satisfy all of the usual theoretical statistical mechanical requirements, except for time reversal symmetry itself. 


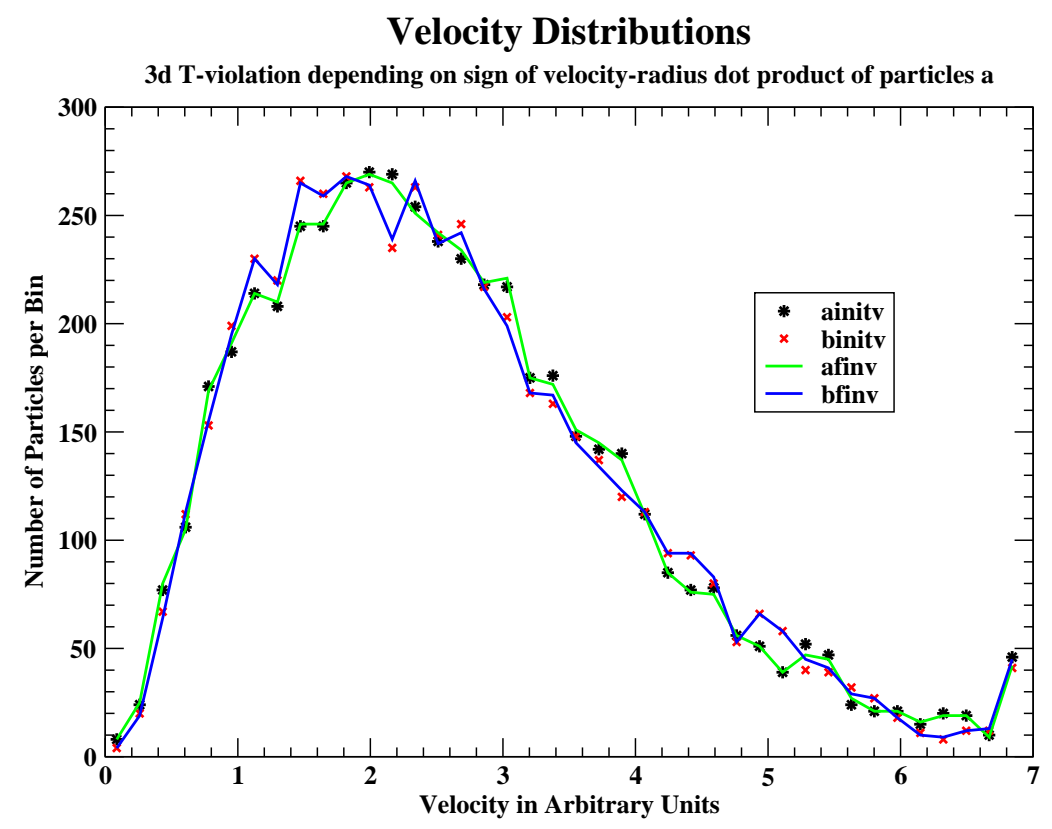

FIG. 5: (Color online) T-violating distributions when the scattering depends on the sign of the dot product of the velocity of one species with its radial vector. The initial (ainitv, binitv) and final (afinv, bfinv) velocity distributions for both species show no significant deviation from Boltzmann.

\section{A KNOWN EXAMPLE}

The neutral kaons are a system of hadronic particles known to be subject to T-violating interactions. Neutral kaons and anti-kaons decay to different final states, but with differing partial rates that would be identical if there were no T-violation. The total decay rates must be equal by the CPT theorem. However, if these particles are contained in a closed volume, so that the decay particles must recombine to form the parent particles then, starting from an initial state containing equal numbers of each kind of particle, a net excess of kaons over anti-kaons (or vice versa) will develop over time due to T-violation. Thus, time-reversal violation produces a matter-antimatter asymmetry, albeit in mesons, not baryons. A recently reported result from FermiLab on the analogous process for B and anti-B mesons decaying to muons [7] finds the number of pairs of positive muons to differ from that of negative pairs produced from B-anti-B meson pairs. This T-violating process could be relevant to the matter-antimatter asymmetry produced in the early Universe. 


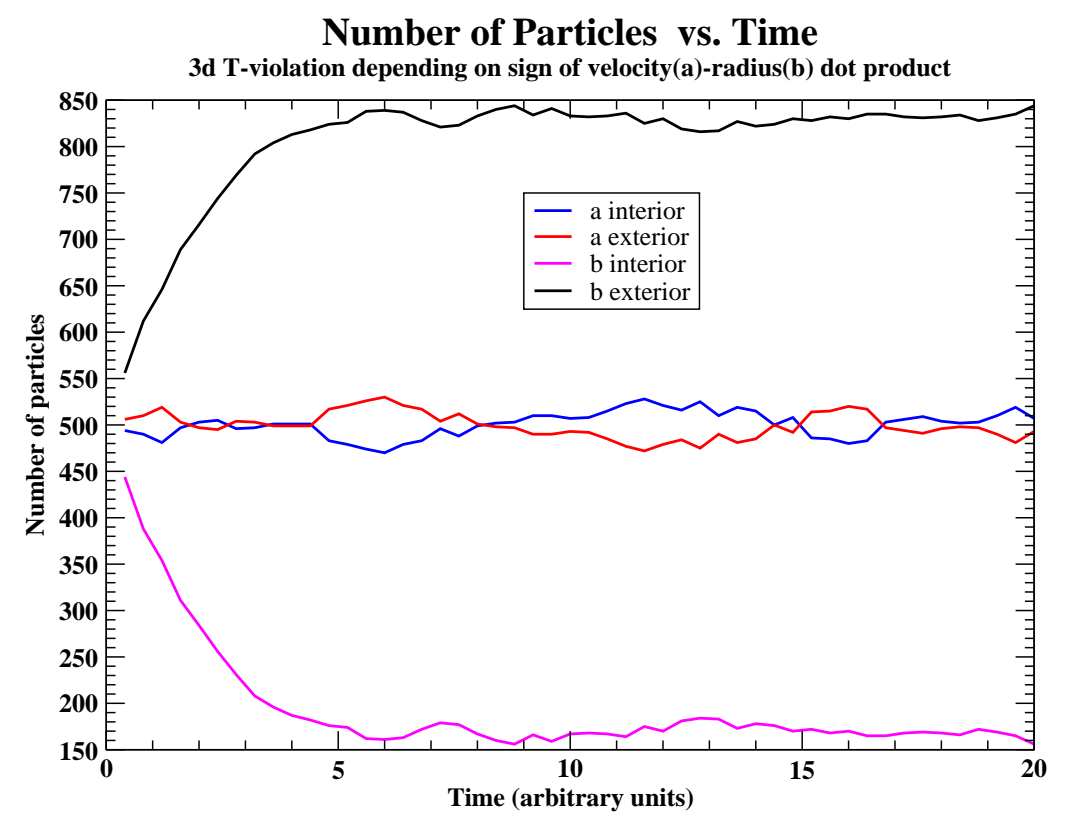

FIG. 6: (Color online) Time dependence of T-violating distributions when the scattering depends on the sign of the dot product of the velocity of one species (a) with the radial vector of the other (b).

\section{A POSSIBLE APPLICATION TO THE EARLY UNIVERSE}

The Universe is often assumed to begin in an organized, low entropy state, with entropy increasing as the Universe evolves. But how could such an organized state have come into being in the first place? Extrapolating from the calculations above, we speculate that, with time reversal violating forces acting for a time prior to the onset of the Big Bang, an initially high entropy state can be transformed into an organized, low entropy state by the time expansion of the Universe commences. We note that recently Gurzadyan and Penrose [8] have used WMAP data to argue for evidence for the existence of a high entropy state occurring "before the Big Bang", as we are assuming here.

We mention several mechanisms by which the formation of an organized, low entropy state could occur. First we consider a physical system with a fourth spatial dimension and imagine that the 3-dimensional world (3-brane) of our visible Universe is the surface of a 4-sphere in that larger space. Starting from a well mixed (high entropy) distribution of, say, matter 
and antimatter over a restricted range in the 4-radial direction, and with the time reversal violating forces acting along that line, we infer on the basis of the calculations presented above (recall Fig 2) that the system will separate into parts with matter on one 3-brane and antimatter on a distinct 3-brane. In other words, the system evolves from what would normally be described as a high entropy (well-mixed) state to one of lower entropy and high density on our 3-brane, which state would then expand our 3-brane following standard cosmology.

Alternatively, and more conventionally, we can imagine that in every small volume of the early Universe, elastic T-violating scattering in the radial direction separates matter and antimatter, again as in our 1-dimensional example calculation. Now as the Universe expands, these separated clumps may remain separated rather than remix. This leads to a picture where there need not be a matter asymmetry in the Universe, but where the matterantimatter annihilation radiation is suppressed by the growth in size of the distinct regions of matter and of antimatter.

Sakharov[9] has discussed the development of excess baryon number in the evolution of the early Universe. His analysis requires three elements: baryon number violation, a nonequilibrium state, and time reversal violation. The first is obvious; regarding the second, we raise the question of how a state of thermodynamic equilibrium should be defined in the presence of $\mathrm{P}$ - and T-violating dynamics and the last we view as the essential element, noting that an excess of baryons over anti-baryons represents a more ordered state than does equality. Thus, this too may be viewed as an example of the development of a more ordered state (by conventional definition) due to a violation of time reversal invariance.

Thus, we observe that time-reversal violation may obviate two of the conditions assumed in Sakharov's discussion [9] of the matter/antimatter problem: Our 5-dimensional system example is in what would normally be considered equilibrium, but nonetheless develops structure and additionally separates matter and antimatter, absent even a baryon-number violating interaction. The only thing that we have maintained is the requirement of $\mathrm{T}$ (equivalently $\mathrm{CP}$ ) violation.

Another mechanism[10] describes a possible origin of our Universe as the white hole arising from an Einstein-Rosen bridge connected to a Weyl metric black hole in another Universe. Note that at the event horizon, the space and time coordinates are interchanged since the 
metric signature for each changes sign; the continuation of what would have been space in a Schwarzchild black hole metric is converted (essentially by the equivalent of coordinate inversion in a sphere) to an infinite time future, and correspondingly time into space.

Two significant points follow: Since all of the material being transported from the original Universe to ours crosses the same radial coordinate value, all of that material appears to have originated in our Universe at a common initial time, at a high initial density and temperature, corresponding to a low entropy state. In fact, since the proper radial velocity becomes infinite, the motion of the particles entering the (new) Universe is aligned in flow (up to quantum fluctuations not considered here) so that the initial state must be viewed as maximally organized, i.e., it has minimum entropy. Secondly, since time reversal is violated, the excess of baryons over anti-baryons in our Universe can be due to an initial condition, namely the in-falling baryon content. Thus only one of Sakharov's requirements, T-violation, remains essential.

\section{CONCLUSION}

The ideas discussed here have been preceded by more general considerations of Dolgov and Zeldovich[11] and by Cohen and Kaplan[12], who also explored the limits of the conventional assumptions. Additionally, Bertolami et al.,13] have shown how CPT violation in the expanding Universe defines a chemical potential so that a baryon asymmetry may develop while in a state of conventional thermal equilibrium. In fact, in this scenario, the asymmetry is so large that it must be diluted by electroweak sphaleron effects to match observation. In any event, it also produces an organized state from a disorganized one, as we suggest here. We have illustrated how more ordered states may evolve from less ordered ones when timereversal-violating interactions are active. Thus, with the conventional definition of entropy, time-reversal-violating interactions can produce reductions in entropy in closed systems. This implies that the ordered state of the early Universe and the observed baryon asymmetry may have been produced by time-reversal-violating interactions. We raise the question of whether or not the conventional definition of entropy requires revision to account for the inaccessibility of some phase space states in the absence of time-reversal-invariance. 


\section{ACKNOWLEDGMENTS}

This work was carried out in part under the auspices of the National Nuclear Security Administration of the U.S. Department of Energy at Los Alamos National Laboratory under Contract No. DE-AC52-06NA25396. We have benefitted from discussions on this topic with Harvey Rose, Cynthia Olsen Reichardt, Charles Reichardt, Daniel Holz and Salman Habib.

[1] LEE T.D. , Int. J. Mod. Phys.A16 (2001) 3633; see Sec. 7.

[2] WAN M.B. , OLSEN REICHHARDT C.J. , NUSSINOV Z. and REICHHARDT C. , Phys. Rev. Lett. 101 (2008) 018102.

[3] STREATER R.F. and WIGHTMAN A.S. , PCT, Spin 8 Statistics, and All That, (W.A. Benjamin, Inc., New York) 1964 (Lib. Congr. Cat. No. 63-22797).

[4] KOSTELECKY V.A. and SAMUEL S. , Phys. Rev. D 39 (1989) 683. For additional and more recent references, see KOSTELECKY V.A. in "Proc. of the 4 th Meeting on CPT and Lorentz Symmety", 8-11 Aug. 2007, Bloomington, USA, edited by KOSTELECKY V.A. (World Scientific, Singapore) 2008, p. 50 (ISBN-13 978-981-277-950-2).

[5] PRICE G.N. , BANNERMAN S.T. , VIERING K. , NAREVICIUS E. and RAIZEN M.G., Phys. Rev. Lett. 100 (2008) 093004.

[6] CHAndRASEKHAR S. , Astrophys. J. 102 (1945) 223. See also, KOBE D.H. , Phys. Rev. AA19 (1979) 205, with a radial vector potential.

[7] WILLIAMS M.R.J. and the D0 collaboration, "Evidence for an anomalous like-sign dimuon charge asymmetry", arXiv:1106.4768v1.

[8] GURZADYAN V.G. and PENROSE R. , "Concentric circles in WMAP data may provide evidence of violent pre-Big-Bang activity", arXiv:1011.3706. See also, PENROSE R. , Cycles of Time: An Extraordinary New View of the Universe, (Bodley Head, London) 2010 (ISBN 9780224080361).

[9] SAKHAROV A.D., Pisma Zh. Eksp. Teor. Fiz. 5(1967) 32 [Sov. Phys. JETP Lett. 5 (1967) $24]$.

[10] PATHRIA R. K. , Nature 240 (1972) 298.

[11] DOLGOV A.D. and ZELDOviCH Y.B., Rev. Mod. Phys. 53 (1981) 1. 
[12] COHEn A.G. and KAPLAN D.B., Phys. Lett. B215 (1988) 67; Nucl. Phys. B 308 (1988) 913.

[13] Bertolami O., COlladAy D. , KOstelecky V.A. and POTting, R., Phys. Lett. B395 (1997) 178. 